Research Article

\title{
Integral-Type Fractional Equations with a Proportional Riemann-Liouville Derivative
}

\author{
Nabil Mlaiki iD \\ Department of Mathematics and General Sciences, Prince Sultan University Riyadh, Riyadh 11586, Saudi Arabia \\ Correspondence should be addressed to Nabil Mlaiki; nmlaiki@psu.edu.sa \\ Received 9 March 2021; Accepted 11 June 2021; Published 23 June 2021 \\ Academic Editor: Antonio Di Crescenzo \\ Copyright (C) 2021 Nabil Mlaiki. This is an open access article distributed under the Creative Commons Attribution License, which \\ permits unrestricted use, distribution, and reproduction in any medium, provided the original work is properly cited. \\ In this paper, we present the necessary conditions where integral-type fractional equations with a proportional Riemann-Liouville \\ derivative have a unique solution. Also, we give an example to illustrate our work.
}

\section{Introduction}

Lately, many researchers have been focusing on the study of various types of fractional problems; we refer the reader to [1-17]. The fixed point and the monotone iterative techniques can be very useful tools to prove the existence and uniqueness of a solution to this type of problems; see [1]. In this manuscript, inspired by the work of Jankowski in [1], we investigate the existence and uniqueness of a solution to the following problem:

$$
\begin{aligned}
D^{\alpha, \rho} \xi(t) & =g\left(t, \xi(t), \int_{0}^{t} \mathscr{K}(t, \tau) \xi(\tau) \mathrm{d} \tau\right) \equiv \mathscr{F} \xi(t), \quad t \in J_{0}=(0, a] ; a>0 \\
\tilde{\xi}(0) & =p
\end{aligned}
$$

where $D^{\alpha, \rho} \xi(t)$ denotes a proportional Riemann-Liouville fractional derivative for $\rho \in[0,1]$ and $0<\alpha<1$. Also, $g \in C(J \times \mathbb{R} \times \mathbb{R}, \mathbb{R}), J=[0, a], \quad$ and $\widetilde{\xi}(0)=t^{1-\alpha} e^{(\rho-1 / \rho) t}$ $\xi(t)_{t \rightarrow 0+}$. Now, we remind the reader of the definition of the proportional Riemann-Liouville fractional integral and derivative.

Definition 1 (see [18]). Let $\alpha \in \mathbb{C} ; \operatorname{Re}(\alpha) \geq 0,0<\rho \leq 1$, and $t>0$.

(i) The following integral is called the proportional Riemann-Liouville fractional integral:

$$
I^{\alpha, \rho} f(t):=\frac{1}{\rho^{\alpha} \Gamma(\alpha)} \int_{0}^{t} e^{[\rho-1 / \rho](t-\tau)}(t-\tau)^{\alpha-1} f(\tau) \mathrm{d} \tau .
$$

(ii) The following derivative is called the proportional Riemann-Liouville fractional derivative:

$$
\begin{aligned}
\left(D^{\alpha, \rho} f\right)(t) & =D^{n, \rho} I^{n-\alpha, \rho} f(t) \\
& =\frac{D_{t}^{n, \rho}}{\rho^{n-\alpha} \Gamma(n-\alpha)} \int_{0}^{t} e^{[\rho-1 / \rho](t-\tau)}(t-\tau)^{n-\alpha-1} f(\tau) \mathrm{d} \tau,
\end{aligned}
$$


where $n=[\operatorname{Re}(\alpha)]+1$ and $D_{t}^{1, \rho}=(1-\rho) f(t)+\rho f^{\prime}(t)$.

Next, we present the following proposition.

Proposition 1 (see [18]). If $\alpha, \gamma \in \mathbb{C}$, where $\operatorname{Re}(\alpha)>0$ and $\operatorname{Re}(\gamma)>0$, then for any $0<\rho \leq 1$, we have $I^{\alpha, \rho}\left(t^{\gamma-1} e^{(\rho-1 / \rho) t}\right)(x)=\left(\Gamma(\gamma) / \rho^{\alpha} \Gamma(\alpha+\gamma)\right) x^{\gamma+\alpha-1} e^{(\rho-1 / \rho) x}$.

In Section 2, we prove the existence and uniqueness of a solution to problem (1) using the fixed point technique. In Section 3, we prove the existence and uniqueness of a solution to problem (1) using the monotone iterative method. In the conclusion, we present an open question.

\section{Fixed Point Approach}

First of all, let $C_{1-\alpha}(J, \mathbb{R})=\left\{f \in C([0, a), \mathbb{R}) \mid t^{1-\alpha}\right.$ $f \in C(J, \mathbb{R})\}$. Now, define the following two weighted norms:

$$
\begin{aligned}
& \|f\|^{*}=\max _{[0, a]} t^{1-\alpha}|f(t)|, \\
& \|f\|_{*}=\max _{[0, a]} t^{1-\alpha} e^{-\lambda t}|f(t)| \text { for fixed } \lambda>0 .
\end{aligned}
$$

Theorem 1. Let $0<\alpha<1,0<\rho \leq 1$, and $g \in C(J \times$ $\mathbb{R} \times \mathbb{R}, \mathbb{R}), \mathscr{K} \in C(J \times J, \times \mathbb{R})$. Let $\beta:=(\rho-1 / \rho)$. Also, assume the following two hypotheses:

(1) There exist nonnegative constants $H, V$, and $W$ such that $|\mathscr{K}(t, s)|<H$ and

$$
\left|g\left(t, u_{1}, u_{2}\right)-g\left(t, v_{1}, v_{2}\right)\right| \leq V\left|v_{1}-u_{1}\right|+W\left|v_{2}-u_{2}\right| \text {. }
$$

(2) $b \equiv\left(a^{\alpha} / \Gamma(2 \alpha) \rho^{\alpha}\right) \quad[V+(H W a / 2 \alpha)]<1$, for $\alpha \epsilon$ $(0,(1 / 2)]$.

Then, initial value problem (1) has a unique solution.

Proof. First, let $S \xi(t)=t^{1-\alpha} e^{(\rho-1 / \rho) t} p+\left(1 / \rho^{\alpha} \Gamma(\alpha)\right) \int_{0}^{t}$ $e^{[(\rho-1 / \rho)(t-\tau)]}(t-\tau)^{\alpha-1} \mathscr{F} \xi(\tau) \mathrm{d} \tau$. Note that if $S$ has a unique fixed point and that is $S \xi(t)=\xi(t)$, then initial value problem (1) has a unique solution, i. e., it will be enough to show that $S$ is a contraction map. So, let $\xi, y \in C_{1-\alpha}(J, \mathbb{R})$; we have two cases:

Case 1: $\alpha \in(0,(1 / 2)]$.

$$
\begin{aligned}
S \xi(t) & =t^{1-\alpha} e^{\beta t} p+\frac{1}{\rho^{\alpha} \Gamma(\alpha)} \int_{0}^{t} e^{\beta(t-\tau)}(t-\tau)^{\alpha-1} \mathscr{F} \xi(\tau) \mathrm{d} \tau \\
\|S \xi-S \mathscr{Y}\|^{*} & =\frac{1}{\rho^{\alpha} \Gamma(\alpha)} \max _{t \in J} t^{1-\alpha} \int_{0}^{t} e^{\beta(t-\tau)}(t-\tau)^{\alpha-1}|\mathscr{F} \xi(\tau)-\mathscr{F} \mathrm{Y}(\tau)| \mathrm{d} \tau \\
& \leq \frac{1}{\rho^{\alpha} \Gamma(\alpha)} \max _{t \in J} t^{1-\alpha}\left\{\int_{0}^{t} e^{\beta(t-\tau)}(t-\tau)^{\alpha-1}\left[+W \int_{0}^{\tau}|\| \mathscr{K}(t, s) \xi(s)-\mathscr{Y}(s)| \mathrm{d} s\right] \mathrm{d} \tau\right\} \\
& \leq \frac{1}{\rho^{\alpha} \Gamma(\alpha)}\|\xi-\mathscr{Y}\|^{*} \max _{t \in J}\left\{t^{1-\alpha} \int_{0}^{t} e^{\beta(t-\tau)}(t-\tau)^{\alpha-1}\left[V \tau^{\alpha-1}+H W \int_{0}^{\tau} s^{\alpha-1} \mathrm{~d} s\right] \mathrm{d} \tau\right\} \\
& =\frac{1}{\rho^{\alpha} \Gamma(\alpha)}\|\xi-\mathscr{Y}\|^{*} \max _{t \in J} t^{1-\alpha} \int_{0}^{t} e^{\beta(t-\tau)}(t-\tau)^{\alpha-1}\left[V \tau^{\alpha-1}+H W \frac{\tau^{\alpha}}{\alpha}\right] \mathrm{d} \tau \\
& \leq \frac{1}{\rho^{\alpha} \Gamma(\alpha)}\|\xi-\mathscr{Y}\|^{*} \max _{t \in J} t^{1-\alpha} e^{-\beta t} \int_{0}^{t} e^{\beta(t-\tau)}(t-\tau)^{\alpha-1}\left[V e^{\beta \tau} \tau^{\alpha-1}+H W e^{\beta \tau} \frac{\tau^{\alpha}}{\alpha}\right] \mathrm{d} \tau \\
& =\frac{1}{\rho^{\alpha} \Gamma(\alpha)}\|\xi-\mathscr{Y}\|^{*} \max _{t \in J} t^{1-\alpha} e^{-\beta t}\left[I^{\alpha, \rho}\left(V t^{\alpha-1} e^{\beta t}\right)+I^{\alpha, \rho}\left(\frac{H W}{\alpha} t^{\alpha} e^{\beta t}\right)\right] \\
& =\frac{\Gamma(\alpha) a^{\alpha}}{\Gamma(\alpha) \Gamma(2 \alpha) \rho^{\alpha}}\left[V+\frac{H W a}{2 \alpha}\|\xi-\mathscr{Y}\|^{*}\right. \\
& =b\|\xi-\mathscr{Y}\|^{*} .
\end{aligned}
$$

Hence, $S$ is a contraction map. Therefore, $S$ has a unique fixed point as desired.

Case 2: $\alpha \in((1 / 2), 1)$; in this case, we use $\|\cdot\|_{*}$ with the positive constant $\lambda>0$ such that

$$
\sqrt{\lambda-\beta}>b_{1} \equiv \frac{e^{-\beta a}(V \alpha+H W a) \Gamma(2 \alpha-1) \sqrt{a^{2 \alpha-1}}}{\alpha \rho^{\alpha} \Gamma(\alpha) \sqrt{\Gamma(2(2 \alpha-1))}} .
$$


It is not difficult to see the following:

(1) $\int_{0}^{t} e^{2(\lambda-\beta) \tau} \mathrm{d} \tau \leq\left(e^{2(\lambda-\beta) t} / 2(\lambda-\beta)\right)$

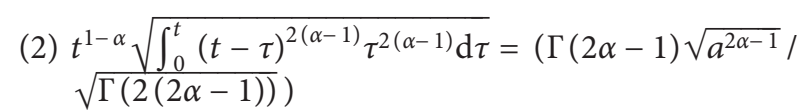

Also, recall the Schwarz inequality for integrals:

$$
\begin{aligned}
\int_{0}^{t}|f(\tau) \| g(\tau)| \mathrm{d} \tau & \leq \sqrt{\int_{0}^{t} f^{2}(\tau) \mathrm{d} \tau} \sqrt{\int_{0}^{t} g^{2}(\tau) \mathrm{d} \tau}, \\
\|S \xi-S \mathscr{Y}\|_{*} & =\frac{1}{\rho^{\alpha} \Gamma(\alpha)} \max _{t \in J} t^{1-\alpha} e^{-\lambda t} \int_{0}^{t} e^{\beta(t-\tau)}(t-\tau)^{\alpha-1}|\mathscr{F} \xi(\tau)-\mathscr{F} \mathrm{Y}(\tau)| \mathrm{d} \tau \\
& \leq \frac{1}{\rho^{\alpha} \Gamma(\alpha)}\|\xi-\mathscr{Y}\|_{*} \max _{t \in J}\left\{t^{1-\alpha} e^{-\lambda t} \int_{0}^{t} e^{\beta(t-\tau)}(t-\tau)^{\alpha-1}\left[V e^{\lambda \tau} \tau^{\alpha-1}\right]+H W e^{\lambda \tau} \int_{0}^{\tau} s^{\alpha-1} \mathrm{~d} s \mathrm{~d} \tau\right\} \\
& \leq \frac{1}{\rho^{\alpha} \Gamma(\alpha)}\|\xi-\mathscr{Y}\|_{*} \max _{t \in J}\left\{t^{1-\alpha} e^{-\lambda t} \int_{0}^{t} e^{\beta(t-\tau)}(t-\tau)^{\alpha-1}\left[V \tau^{\alpha-1} e^{\lambda \tau}+H W \frac{\tau^{\alpha}}{\alpha} e^{\lambda \tau}\right] \mathrm{d} \tau\right\} \\
& \leq \frac{V \alpha+H W a}{\alpha \rho^{\alpha} \Gamma(\alpha)}\|\xi-\mathscr{Y}\|_{*} \max _{t \in J}\left\{t^{1-\alpha} e^{-\lambda t} \int_{0}^{t} e^{\beta(t-\tau)} e^{\lambda \tau}(t-\tau)^{(\alpha-1)} \tau^{\alpha-1} \mathrm{~d} \tau\right\} \\
& =\frac{V \alpha+H W a}{\alpha \rho^{\alpha} \Gamma(\alpha)}\|\xi-\mathscr{Y}\|_{*} \max _{t \in J}\left\{t^{1-\alpha} e^{-\lambda t} \int_{0}^{t} e^{\beta(t-\tau)+\lambda \tau}(t-\tau)^{(\alpha-1)} \tau^{\alpha-1} \mathrm{~d} \tau\right\} \\
& \leq \frac{V \alpha+H W a}{\alpha \rho^{\alpha} \Gamma(\alpha)}\|\xi-\mathscr{Y}\|_{*} \max _{t \in J}\left\{t^{1-\alpha} e^{-\lambda t} \times \sqrt{\int_{0}^{t}(t-\tau)^{2(\alpha-1)} \tau^{2(\alpha-1)} \mathrm{d} \tau} \sqrt{\int_{0}^{t} e^{2 \beta(t-\tau)+2 \lambda \tau} \mathrm{d} \tau}\right\} \\
& \leq \frac{V \alpha+H W a}{\alpha \rho^{\alpha} \Gamma(\alpha)}\|\xi-\mathscr{Y}\|_{*} \max _{t \in J}\left\{t^{1-\alpha} e^{-\lambda t} \times \sqrt{\int_{0}^{t}(t-\tau)^{2(\alpha-1)} \tau^{2(\alpha-1)} \mathrm{d} \tau} \sqrt{\int_{0}^{t} e^{2(\lambda-\beta) \tau} \mathrm{d} \tau}\right\} \\
& \leq \frac{b_{1}}{\sqrt{\lambda-\beta}\|\xi-\mathscr{Y}\|_{*} .}
\end{aligned}
$$

Thus, $S$ is a contraction map. Therefore, $S$ has a unique fixed point as required.

As an application to Theorem 1, consider the following problem:

$$
\begin{aligned}
D^{\alpha, \rho} y(t) & =-L(t) y(t)+\xi(t), \\
\widetilde{\xi}(0) & =r .
\end{aligned}
$$

If

$$
\frac{a^{\alpha}}{\rho^{\alpha} \Gamma(2 \alpha)} \max _{t \in J}|L(t)|<1, \quad \text { for } 0<\alpha \leq \frac{1}{2},
$$

then it is not difficult to see that, by using Theorem 1, problem (9) has a unique solution. In closing of this section, the following linear problem is considered:

$$
\begin{aligned}
D^{\alpha, \rho} \xi(t) & =-L(t) \xi(t)+z(t), \quad t \in J_{0}, \\
\tilde{\xi}(0) & =r .
\end{aligned}
$$

Now, we introduce the following hypothesis.
Hypothesis $1\left(H_{1}\right)$

(1) $L(t)=L, t \in J$ or

(2) The function $L$ is nonconstant on $J$ and

$$
\frac{a^{\alpha}}{\rho^{\alpha} \Gamma(2 \alpha)} \max _{t \in J}|L(t)|<1 \quad \text { only if } \quad \alpha \in\left(0, \frac{1}{2}\right) .
$$

The following lemma is a consequence of Theorem 1.

Lemma 1. If $\alpha \in(0,1), L \in C(J, \mathbb{R}), z \in C_{1-\alpha}(J, \mathbb{R})$, and hypothesis $\left(H_{1}\right)$ holds, then problem (11) has a unique solution.

We would like to bring to the reader's attention that, in [1], in the hypothesis $\rho$ should be as follows: $\rho \equiv$ $\left(T^{q} / \Gamma(2 q)\right)[K+(W L T / 2 q)]$ which he used to prove the case where $q \in(0,(1 / 2)]$. This way, his result will be stronger or he can just change the last equality to the inequality. 


\section{Monotone Iterative Method}

First of all, we start by introducing the following hypothesis.

Hypothesis $2\left(\mathrm{H}_{2}\right)$

(1) $L(t)=L, t \in J$ or

(2) The function $L$ is nonconstant, and if $L(t)$ is negative, then there exists $\bar{L}$ which is nondecreasing, where $-L(t) \leq \bar{L}(t)$ on $J$ and for every $x \in J$, we have

$$
\frac{e^{\beta x}}{\rho^{\alpha} \Gamma(\alpha)} \int_{0}^{a}(a-\tau)^{\alpha-1} e^{\beta(a-\tau)} \bar{L}(\tau) \mathrm{d} \tau<1
$$
lemma.
Lemma 2. Let $\alpha \in(0,1)$ and $L \in C(J,[0, \infty))$ or $L \in C(J,(-\infty, 0])$. Also, denote by $\beta:=(\rho-1 / \rho)$. Assume that $q \in C_{1-\alpha}(J, \mathbb{R})$ is a solution to the following problem:

$$
\begin{aligned}
D^{\alpha, \rho} q(t) & \leq-L(t) q(t), \quad t \in J_{0}, \\
\widetilde{q}(0) & <0 .
\end{aligned}
$$

If $\left(\mathrm{H}_{2}\right)$ holds, then $q(t) \leq 0$ for all $t \in J$.

Proof. Assume that our lemma is false, that is, there exist $x, y \in[0, a)$ such that $q(x)=0, q(y)>0$, and $q(t) \leq 0$ for $t \in(0, x] ; q(t)>0$ for $t \in(x, y]$. Let $x_{0}$ be the first maximal point of $q$ on $[x, y]$.

Case 1: assume that $L(t) \geq 0$ for all $t \in J$. Thus, $D^{\alpha, \rho} q(t) \leq 0$ for $t \in[x, y]$. Hence,

$$
\int_{x}^{x_{0}} D^{\alpha, \rho} q(t) \leq 0
$$

Therefore, $B \equiv I^{\rho, 1-\alpha} q\left(x_{0}\right)-I^{\rho, 1-\alpha} q(x) \leq 0$, but

$$
\begin{aligned}
B & =\frac{1}{\rho^{1-\alpha} \Gamma(1-\alpha)}\left[\int_{0}^{x_{0}} e^{\beta\left(x_{0}-\tau\right)}\left(x_{0}-\tau\right)^{-\alpha} q(\tau) \mathrm{d} \tau-\int_{0}^{x} e^{\beta(x-\tau)}(x-\tau)^{-\alpha} q(\tau) \mathrm{d} \tau\right] \\
& =\frac{1}{\rho^{1-\alpha} \Gamma(1-\alpha)}\left\{\int_{0}^{x}\left[e^{\beta\left(x_{0}-\tau\right)}\left(x_{0}-\tau\right)^{-\alpha}-e^{\beta(x-\tau)}(x-\tau)^{-\alpha}\right] q(\tau) \mathrm{d} \tau+\int_{x}^{x_{0}} e^{\beta\left(x_{0}-\tau\right)}\left(x_{0}-\tau\right)^{-\alpha} q(\tau) \mathrm{d} \tau\right\} \\
& >\frac{1}{\rho^{1-\alpha} \Gamma(1-\alpha)} \int_{x}^{x_{0}} e^{\beta\left(x_{0}-\tau\right)}\left(x_{0}-\tau\right)^{-\alpha} q(\tau) \mathrm{d} \tau>0,
\end{aligned}
$$

which leads us to a contradiction given the fact that $B \leq 0$.

Case 2: assume that $L(t) \leq 0$ for all $t \in J$, and consider $\widetilde{L}$ to be nondecreasing on $J$. Now, if we apply $I^{\alpha, \rho}$ on problem (14), we obtain

$$
q(t)-\widetilde{q}(0) \frac{e^{\beta t} t^{\alpha-1}}{\rho^{\alpha-1} \Gamma(\alpha)} \leq-I^{\alpha, \rho}[L(t) q(t)], \quad \text { for } t \in\left[x, x_{0}\right]
$$

Notice that $\widetilde{q}(0)\left(e^{\beta t} t^{\alpha-1} / \rho^{\alpha-1} \Gamma(\alpha)\right) \leq 0$ which is due to the fact that $\tilde{q}(0) \leq 0$. Thus, 


$$
\begin{aligned}
q\left(x_{0}\right) & \leq-\frac{1}{\rho^{\alpha} \Gamma(\alpha)} \int_{0}^{x_{0}}\left(x_{0}-\tau\right)^{\alpha-1} e^{\beta\left(x_{0}-\tau\right)} L(\tau) q(\tau) \mathrm{d} \tau \\
& =-\frac{1}{\rho^{\alpha} \Gamma(\alpha)}\left[\int_{0}^{x_{0}}\left(x_{0}-\tau\right)^{\alpha-1} e^{\beta\left(x_{0}-\tau\right)} L(\tau) q(\tau) \mathrm{d} \tau+\int_{x}^{x_{0}}\left(x_{0}-\tau\right)^{\alpha-1} e^{\beta\left(x_{0}-\tau\right)} L(\tau) q(\tau) \mathrm{d} \tau\right] \\
& <-\frac{q\left(x_{0}\right)}{\rho^{\alpha} \Gamma(\alpha)} \int_{0}^{x_{0}}\left(x_{0}-\tau\right)^{\alpha-1} e^{\beta\left(x_{0}-\tau\right)} L(\tau) \mathrm{d} \tau, \text { let } \sigma=\frac{\tau}{x_{0}} \\
& =-\frac{q\left(x_{0}\right) e^{\beta x_{0}} x_{0}^{\alpha}}{\rho^{\alpha} \Gamma(\alpha)} \int_{0}^{1}(1-\sigma)^{\alpha-1} e^{\beta(1-\sigma)} L\left(\sigma x_{0}\right) \mathrm{d} \sigma \\
& \leq \frac{q\left(x_{0}\right) e^{\beta x_{0}} x_{0}^{\alpha}}{\rho^{\alpha} \Gamma(\alpha)} \int_{0}^{1}(1-\sigma)^{\alpha-1} e^{\beta(1-\sigma)} \widetilde{L}(\sigma a) \mathrm{d} \sigma \\
& =\frac{q\left(x_{0}\right) e^{\beta\left(x_{0}+(1 / a)\right)}}{\rho^{\alpha} \Gamma(\alpha) a^{\alpha}} \int_{0}^{a}(a-\tau)^{\alpha-1} e^{\beta(a-\tau)} \widetilde{L}(\tau) \mathrm{d} \tau \\
& \leq \frac{q\left(x_{0}\right) e^{\beta\left(x_{0}+(1 / a)\right)}}{\rho^{\alpha} \Gamma(\alpha)} \int_{0}^{a}(a-\tau)^{\alpha-1} e^{\beta(a-\tau)} \widetilde{L}(\tau) \mathrm{d} \tau .
\end{aligned}
$$

Hence, $\quad q\left(x_{0}\right)\left[1-\left(e^{\beta\left(x_{0}+(1 / a)\right)} / \quad \rho^{\alpha} \Gamma(\alpha)\right) \int_{0}^{a}(a-\tau)^{\alpha-1}\right.$ $\left.e^{\beta(a-\tau)} \widetilde{L}(\tau) \mathrm{d} \tau\right] \leq 0$. Using hypothesis $\left(H_{2}\right)$ implies that $q\left(x_{0}\right) \leq 0$, which leads us to a contradiction, and this concludes our proof.

We say that $y$ is a lower solution of problem (1) if

$$
D^{\alpha, \rho} y(t) \leq \mathscr{F} y(t), \quad t \in J_{0}, \widetilde{y}(0) \leq 0,
$$

$$
\left|g\left(t, u_{1}, u_{2}\right)-g\left(t, v_{1}, v_{2}\right)\right| \leq L(t)\left|v_{1}-u_{1}\right| \quad \text { whenever } x_{0} \leq u_{1} \leq v_{1} \leq y_{0} \text { and } u_{2} \leq v_{2} \text {. }
$$

Theorem 2. Assume that $x_{0}$ is a lower solution of problem (1) and $y_{0}$ is an upper solution of problem (1), where $x_{0}, y_{0} \in C_{1-\alpha}(J, \mathbb{R})$. Moreover, assume that hypotheses $H_{1}, H_{2}$, and $H_{3}$ hold; problem (1) has solutions in $\left[x_{0}, y_{0}\right]=$ $\left\{y \in C_{1-\alpha}(J, \mathbb{R}) \mid x_{0}(t) \leq y(t) \leq y_{0}(t), t \in J_{0}, \widetilde{x}_{0}(0) \leq \widetilde{y}(0) \leq\right.$ $\left.\widetilde{y}_{0}(0)\right\}$.

Proof. Using Lemmas 1 and 2, the proof is similar to the proof of Theorem 2 in [1].

Now, we present the following example. and we say that $y$ is an upper solution of problem (1) if

$$
D^{\alpha, \rho} y(t) \geq \mathscr{F} y(t), \quad t \in J_{0}, \tilde{y}(0) \leq 0 .
$$

Next, the following hypothesis is defined.

Hypothesis 3. $\left(\mathrm{H}_{3}\right)$. There exists a function $L \in C(J, \mathbb{R})$ where

Example 1. Let $0<\alpha<1, \quad 0<\rho \leq 1, \quad \beta=(\rho-1 / \rho)$, and $\mathbb{A}, \mathbb{B} \in C([0,1],(0, \infty))$ such that $\mathbb{A}(t) \leq \mathbb{B}(t)$ for $t \in[0,1]$. Now, consider the following problem:

$$
\begin{aligned}
D^{\alpha, \rho} \xi(t) & \equiv \mathscr{F} \xi(t), \quad t \in J_{0}=(0,1], \\
\tilde{\xi}(0) & =0,
\end{aligned}
$$

where

$$
\mathscr{F} \xi(t)=\frac{\rho^{\alpha} e^{\beta t} t^{1-\alpha}}{\Gamma(2-\alpha)}+\mathbb{A}(t)\left[t e^{\beta t}-1-\xi(t)\right]^{3}+\frac{\beta}{e^{\beta t}-1} \mathbb{B}(t) \int_{0}^{t}[\sin (t \tau)]^{4} \xi(\tau) \mathrm{d} \tau .
$$


Now, let $x_{0}(t)=0$ and $y_{0}(t)=t e^{\beta t}$; first, note that $x_{0}(t)$ is a lower solution of problem (22). Next, we show that $y_{0}(t)$ is an upper solution of problem (22):

$$
\begin{aligned}
\mathscr{F} y_{0}(t) & =\frac{\rho^{\alpha} e^{\beta t} t^{1-\alpha}}{\Gamma(2-\alpha)}-\mathbb{A}(t)+\frac{\beta}{e^{\beta t}-1} \mathbb{B}(t) \int_{0}^{t}[\sin (t \tau)]^{4} \tau e^{\beta \tau} \mathrm{d} \tau \\
& \leq \frac{\rho^{\alpha} e^{\beta t} t^{1-\alpha}}{\Gamma(2-\alpha)}-\mathbb{A}(t)+\frac{\beta}{e^{\beta t}-1} \mathbb{B}(t) \int_{0}^{t} e^{\beta \tau} \mathrm{d} \tau \\
& =\frac{\rho^{\alpha} e^{\beta t} t^{1-\alpha}}{\Gamma(2-\alpha)}-\mathbb{A}(t)+\frac{\beta}{e^{\beta t}-1} \mathbb{B}(t)\left[\frac{e^{\beta t}}{\beta}-\frac{1}{\beta}\right]<\frac{\rho^{\alpha} e^{\beta t} t^{1-\alpha}}{\Gamma(2-\alpha)} \\
& =D^{\alpha, \rho} y_{0}(t) .
\end{aligned}
$$

Thus, $y_{0}(t)$ is an upper solution of problem (22). Now, it is not difficult to see that all the hypotheses of Theorem 2 are satisfied. Therefore, problem (22) has solutions in $\left[x_{0}, y_{0}\right]$ if $\alpha \in((1 / 2), 1)$, and for $\alpha \in(0,(1 / 2)]$, we need to assume that $\left(1 / \rho^{\alpha} \Gamma(2 \alpha)\right) \max _{t \in[0,1]}|\mathbb{A}(t)|<1$.

\section{Conclusion}

In closing, note that the results of Jankowski [1] are a special case of our work which is by taking $\rho=1$. Also, we would like to bring to the reader attention the following open question.

What are the necessary and sufficient conditions for problem (1) to have a unique solution if $\rho$ is not constant, but it is a function of $\$ \mathrm{t} \$$ say $g(t)$, so that the problem involves $D^{\alpha, g(t)}$ ?

\section{Data Availability}

No data were used to support this study.

\section{Conflicts of Interest}

The author declares that there are no conflicts of interest.

\section{Acknowledgments}

The author would like to thank Prince Sultan University for funding this work through the research group Nonlinear Analysis Methods in Applied Mathematics (NAMAM) (group no. RG-DES-2017-01-17).

\section{References}

[1] T. Jankowski, "Fractional equations of Volterra type involving a Riemann-Liouville derivative," Applied Mathematics Letters, vol. 26, no. 3, pp. 344-350, 2013.

[2] T. Jankowski, "Fractional differential equations with deviating arguments," Dynamic Systems and Applications, vol. 17, pp. 677-684, 2008.
[3] V. Lakshmikantham, S. Leela, and J. Vasundhara, Theory of Fractional Dynamic Systems, Cambridge Academic Publishers, Cambridge, UK, 2009.

[4] L. Lin, X. Liu, and H. Fang, "Method of upper and lower solutions for fractional differential equations," Electronic Journal of Differential Equations, vol. 100, pp. 1-13, 2012.

[5] Q. M. Al-Mdallal, "Monotone iterative sequences for nonlinear integro-differential equations of second order," Nonlinear Analysis: Real World Applications, vol. 12, no. 6, pp. 3665-3673, 2011.

[6] Q. M. Al-Mdallal, "Boundary value problems for nonlinear fractional integro-differential equations: theoretical and numerical results," Advances in Difference Equations, vol. 18, no. 1, pp. 1-13, 2012.

[7] M. Syam, Q. Al-Mdallal, and M. Anwar, "An efficient numerical algorithm for solving fractional higher-order nonlinear integrodifferential equations," Abstract and Applied Analysis, vol. 2015, Article ID 616438, 2015.

[8] F. A. McRae, "Monotone iterative technique and existence results for fractional differential equations," Nonlinear Analysis: Theory, Methods \& Applications, vol. 71, no. 12, pp. 6093-6096, 2009.

[9] I. Podlubny, Fractional Differential Equations, Academic Press, San Diego, CA, USA, 1999.

[10] J. D. Ramírez and A. S. Vatsala, "Monotone iterative technique for fractional differential equations with periodic boundary conditions," Opuscula Mathematica, vol. 29, no. 3, pp. 289-304, 2009.

[11] Z. Wei, Q. Li, and J. Che, "Initial value problems for fractional differential equations involving Riemann-Liouville sequential fractional derivative," Journal of Mathematical Analysis and Applications, vol. 367, no. 1, pp. 260-272, 2010.

[12] G. Wang, "Monotone iterative technique for boundary value problems of a nonlinear fractional differential equation with deviating arguments," Journal of Computational and Applied Mathematics, vol. 236, no. 9, pp. 2425-2430, 2012.

[13] G. Wang, R. P. Agarwal, and A. Cabada, "Existence results and the monotone iterative technique for systems of nonlinear fractional differential equations," Applied Mathematics Letters, vol. 25, no. 6, pp. 1019-1024, 2012.

[14] S. Zhang, "Monotone iterative method for initial value problem involving Riemann-Liouville fractional derivatives," Nonlinear Analysis: Theory, Methods \& Applications, vol. 71, no. 5-6, pp. 2087-2093, 2009. 
[15] E. Deniz, A. O. Akdemir, and E. Yüksel, "New extensions of Chebyshev-Pòlya-Szegö type inequalities via conformable integrals," AIMS Mathematics, vol. 4, no. 6, pp. 1684-1697, 2019.

[16] M. K. Sajid, R. S. Ali, and I. Nayab, "Some results of generalized k-fractional integral operator with k-bessel function," Turkish Journal of Science, vol. 5, no. 3, pp. 157-169, 2020.

[17] A. Ekinci and M. E. Özdemir, "Some new integral inequalities via Riemann liouville integral operators," Applied and Computational Mathematics, vol. 3, no. 18, pp. 288-295, 2019.

[18] F. Jarad, M. A. Alqudah, and T. Abdeljawad, "On more general forms of proportional fractional operators," Open Mathematics, vol. 18, no. 1, pp. 167-176, 2020. 